\title{
CIENCIA Y DOCENCIA EN AGUSTÍN Y TOMÁS DE AQUINO (DEL MAESTRO AGUSTINIANO AL MAESTRO TOMISTA)'
}

\author{
Antonio Pérez-Estévez \\ Universidad del Zulia. Venezuela
}

\section{RESUMEN}

Se hace un análisis comparativo de los conceptos de ciencia, maestro y discípulo tal como aparecen en el De Magistro de san Agustín y en la cuestión 11, «De Magistro», de la disputación De Veritate de santo Tomás de Aquino.

La ciencia agustiniana de las verdades inteligibles y eternas se adquire por una visión mental del entendimiento y la razón y supone la buena voluntad del sujeto; la tomista consiste más bien en el conjunto de formas inteligibles adquiridas a partir de un proceso deductivo, lógico-necesario, que se inicia con los primeros principios y axiomas evidentes. Para Agustín, hay sólo un maestro de las verdades inteligibles que se encuentra en el interior de cada alma racional y que se manifiesta sólo al hombre interior o aquel en el que domina la buena voluntad. Para Tomás de Aquino, existen dos maestros: uno principal e interior, que es Dios, y otro secundario y externo, pero también maestro, el cual ayuda al discípulo a adquirir la habilidad y el hábito de deducir verdades a partir de los primeros principios y axiomas evidentes y con él el número de formas inteligibles. Para Agustín, todos somos discípulos del único maestro de la Verdad que es Cristo y seremos buenos o malos discípulos de acuerdo con la buena o mala voluntad que lo domine. Para Tomás de Aquino, el discípulo posee a medias las nociones o verdades que va a aprender, es decir, en sus primeros principios 0 axiomas evidentes y adquirirá con la ayuda importante del maestro a adquirir la habilidad y el hábito de deducir y desplegar las nociones y verdades encerradas en los primeros principios y axiomas evidentes.

\begin{abstract}
Science and teaching in Augustine and Thomas Aquinas. The author tries to make a comparative analisis of the notions science, teacher and disciple such as they appear in St. Augustine's De Magistro and in question 11th, «De Magistro», of the Disputatio De Veritate of Saint Thomas Aquinas.
\end{abstract}

1 El texto latino utilizado en este trabajo del De Magistro de San Agustín es el reproducido en Obras de San Agustín, tomo III, edición bilingüe, 3. a edición, Madrid, B.A.C.,1963. El número de las citas remiten a capítulo y párrafo, y siguen la numeración de la edición señalada.

El texto que seguimos del De Magistro de Tomás de Aquino es el reproducido en Quaestiones disputatae, vol. 1, De Veritate, quaestio 11 «De Magistro», Turín-Roma, Marietti, 1964. El número de página citado va acompañado de las letras a y $b$ para significar la primera o la segunda columna de dicha página.

Frecuentemente aparecerá: D.M. = De Magistro de San Agustín; «D.M.» = «De Magistro» de santo Tomás de Aquino. 
Augustinian science of intelligible and eternal truths is adquired by a mental view of intellect and reason; it supposes the good will of knowing subject. Thomist science consists of a number of intelligible forms adquired from a deductive logic-necessary process that begins with the first obvious principles and axioms. For Augustin, there is only one teacher of intelligible truths that hides himself in the deepest of every rational soul and shows the Truth only to the interior man, that is, the one dominated by the good will. For Thomas Aquinas, there exist two different teachers: the main and interior one that is God, and another external and human, with perfect and actual knowledge, who helps the disciple to get the ability and the habit of deducing notions and truths from the first obvious principles and axioms. For Augustin, we all are disciples of the unique teacher of Truth, Christ, and we will be good or bad disciples according to our good or evil will. For Thomas Aquinas, every disciple has from the very beginning all the notions and truths that he is going to learn, but in potency or in their seminal reasons - the first obvious principles and axioms-; but he will try, with the important help of the teacher, to get the ability and the habit of deducing and developping all notions and truths enclosed within the first evident principles and axioms.

\section{CIENCIA Y DOCENCIA EN SAN AGUSTÍN}

\subsection{Docencia y palabra}

San Agustín, siguiendo los pasos de Platón, confía poco en el poder de la palabra para vehicular ideas y servir como instrumento en el proceso de enseñanza-aprendizaje. Nada podemos aprender por medio de aquellos signos que llamamos palabras, dice textualmente Agustín en un texto del De Magistro (10,34). De hecho, Agustín considera que nada podemos aprender por medio de signos en general, «nihil invenies quod per sua signa discatur» (D.M., 10,33). Y esto se debe a que los signos verbales, antes de ser signos, es decir, antes de que signifiquen alguna cosa, son un conjunto de sonidos sin sentido que afectan nuestros oídos; este conjunto de sonidos se convierte en signos justamente cuando conocemos la cosa a la que estos signos apuntan o significan. En el signo descubrimos, dice Agustín, dos cosas distintas: el sonido y el significado: el sonido no lo percibimos por el signo sino inmediatamente por el oído afectado; la significación por la visión de la cosa significada. ${ }^{2}$ Un signo es signo sólo cuando ya conocemos por la visión la cosa significada; de lo contrario, no pasa de ser un conjunto de sonidos o estrépito de palabras que afecta nuestros oídos; el signo supone el conocimiento previo de la cosa significada.

Aprender es conocer algo nuevo, y, si nadie aprende nada por medio de signos y palabras, significa que no podemos adquirir ciencia alguna por medio del lenguaje oído: «verbis auditis nec verba discuntur» (D.M., 11,36), por las palabras oídas no aprendemos ni siquiera palabras, dice Agustín, extremando la imposibilidad de aprender algo con las palabras. El maestro, para Agustín, no puede hacer que el conocimiento del discípulo aumente, es decir, aprenda por medio de palabras ni de signos.

\subsection{Ciencia y visión}

«Nos non posse nisi visa cognoscere» (D.M., 11,37). No podemos conocer sino lo que vemos.

«Discit autem nullo modo, nisi et ipse quod dicitur videat; ubi iam non verbis quae sonuerunt sed rebus ipsis et sensibus discit» (D.M., 12,39). De ninguna manera aprende si no es viendo lo

2 De Magistro, 10,34: «in signo cum duo sint, sonus et significatio, sonum certe non per signum percipimus, sed eo ipso aure pulsata; significationem autem re, quae significatur, aspecta [...]». 
mismo que dice; y entonces no aprende por medio de las palabras escuchadas sino por medio de las cosas mismas y los sentidos.

Descartados los signos, las palabras y el oído como medios para conocer y adquirir ciencia, Agustín va a afirmar reiteradamente que «sólo aprendemos y conocemos por la visión de las cosas mismas» (D.M., 11,37). La visión inmediata de la cosa es la única manera con la que verdaderamente conocemos y aprendemos la cosa. «Nos non posse nisi visa cognoscere». Y una vez vista y conocida la cosa, podemos señalarla o referirnos a ella por medio de un signo verbal o palabra. Es la visión el sentido fundamental por el que aprendemos y adquirimos ciencia. Es por la presencia inmediata de la cosa a nuestros sentidos o a nuestra mente que conocemos y aprendemos y, por tanto, me enseña. Por eso, Agustín va a definir al maestro de la siguiente manera: me enseña algo aquel que ofrece a mis ojos, a alguno de mis sentidos corporales o a mi mente aquello que quiero conocer, «is me autem aliquid docet, qui vel oculis, vel ulli corporis sensui, vel ipsi etiam menti praebet ea quae cognoscere volo» (D.M., 11,36).

Agustín distingue la ciencia sensible y práctica de la ciencia inteligible. Por la ciencia sensible y práctica aprendemos las cosas del mundo sensible y cómo utilizarlas; a ella accedemos por medio de su percepción y, especialmente, por la vista. Conozco una casa, el mar, un hombre, un perro... cuando los percibo o cuando los veo. Y, una vez conocida una cosa por la percepción o la visión, el conjunto o estrépito de sonidos verbales que afecta nuestro oído puede convertirse en signo y remitir a la cosa significada. El estrépito de sonidos se convierte en palabras con significado preciso sólo cuando conocemos de antemano por medio de la percepción o visión la cosa significada. Sarabellae, cofias o adornos en la cabeza, deja de ser un estrépito de sonidos sin sentido sólo si conocemos de antemano - por la percepción o la visión - lo que es adorno y cabeza. El conocimiento del mundo sensible que utilizamos continuamente en nuestra vida cotidiana, lo alcanzamos por medio de la percepción sensible y, muy especialmente, por medio de la visión de las cosas que nos rodean. Las palabras o signos verbales simplemente nos hacen recordar las cosas ya conocidas por medio de la percepción sensible o visión.

La ciencia inteligible es el conocimiento de las realidades inteligibles a las que accede sólo el hombre interior por el entendimiento y la razón. Pero Agustín, siguiendo a Platón, habla de «visión del entendimiento y de la razón», por medio de la que conoce el mundo interior inteligible y espiritual. El proceso interior del conocimiento inteligible es, para Agustín, semejante y análogo al proceso de conocimiento sensible. Si con la vista descubrimos y conocemos cosas visibles o iluminadas por la luz del sol, con èl entendimiento y razón descubrimos y conocemos cosas iluminadas por una luz inteligible interior. Fuera vemos cosas sensibles; dentro cotemplamos o intuimos realidades mentales, ideas o verdades inteligibles. Puede suceder que alguien, por la debilidad de su visión interior, no pueda contemplar toda una realidad mental o una verdad de una sola vez; en ese caso, se le recomienda contemplar primero las partes de que consta en un proceso de análisis, con el fin de que quede manifiesta la realidad o verdad completa. ${ }^{3}$

3 De Magistro, 12, 40: «Nam quod saepe contingit, ut interrogatus aliquid neget, atque ad id fatendum aliis interrogationibus urgeatur, fit hoc imbecilitate cernentis, qui de re tota illam lucem consulere non potest; quod ut partibus faciat admonetur, cum de iisdem istis partibus interrogatur, quibus illa summa constat quam totam cernere non valebat». 
La luz inteligible interior proviene de la Verdad con la que disfruta y se ilumina el hombre interior, «in illa interiore luce veritatis qua ipse qui dicitur homo interior, illustratur et fruitur [...]» (D.M., 12,40). Esta Verdad va a ser identificada con Cristo, que habita en el hombre interior, es decir, con el poder inconmutable de Dios y con la sabiduría sempiterna, a la que toda alma racional contempla y consulta. Pero, esta Verdad o Sabiduría sempiterna se muestra a cada alma racional en la medida en que ésta pueda captarla de acuerdo con su buena o mala voluntad. ${ }^{4} \mathrm{La}$ ciencia inteligible humana participa de la Verdad o Sabiduría divina en la medida en que, de acuerdo con la buena o mala voluntad del hombre particular, puede captarla. La Sabiduría divina se muestra como luz interior de la Verdad a toda alma racional; pero cada alma racional capta esta Sabiduría divina y participa de ella de acuerdo con la bondad o maldad concretas de la voluntad de cada alma racional. La ciencia de las cosas inteligibles dice una directa relación con la buena o mala voluntad de quien la posee. El sujeto de la ciencia inteligible que posee una buena voluntad estará en mejor disposición de captar la luz interior de la verdad que aquel otro que no posea dicha buena voluntad. La ciencia de las cosas inteligibles está, por tanto, en proporción directa con la buena 0 mala voluntad del sujeto o persona que posee dicha ciencia. Ciencia y virtud van en san Agustín de la mano.

La adquisición de ciencia inteligible entraña en Agustín un proceso paralelo de buena voluntad, es decir, de virtud y santidad que posibilita al hombre recogerse en su interior y captar («capere») cada día con más perspicacia la luz de la Sabiduría sempiterna que se muestra («panditur») en lo más profundo de nuestra alma. La cantidad y la calidad de ciencia inteligible para Agustín depende de la capacidad de cada persona, de acuerdo con su buena o mala voluntad, para recogerse en su «hombre interior», desprendiéndose del hombre exterior o de los sentidos, y contemplar intuitivamente la Luz de la Sabiduría sempiterna y de la virtud inconmutable de Dios. La ciencia inteligible o el conocimiento de la Verdad es el resultado de una rica vida interior, lograda a través del orden y el dominio de las facultades superiores de la mente o del alma sobre los sentidos y las pasiones del cuerpo, establecida por la buena voluntad, tal como describe en el Libro I del $D e$ Libero Arbitrio.

La ciencia inteligible se enraíza en la visión intuitiva de la Verdad sempiterna que es Cristo y que habita en el interior de cada alma racional. De esta sabiduría eterna no existen maestros humanos y por eso se engañan quienes llaman maestros a quienes no lo son, «falluntur homines ut eos qui non sunt magistros vocent» (D.M., 14,45). El único maestro de esta ciencia interior capaz de mostrarnos las verdades inteligibles es Cristo o la Sabiduría y Verdad sempiterna. Y los únicos discípulos de esta Verdad, aquellos capaces de ver interiormente, «quisquis autem cernere potest, intus est discipulus veritatis [...]» (D.M., 13,41).

4 De Magistro, 11,38: «[...] in interiore homine habitare dictus est Christus, id est incommutabilis Dei virtus atque sempiterna sapientia, quam quidem omnia rationalis anima consulit; sed tantum cuique panditur quantum capere propter propriam sive malam sive bonam voluntatem potest». 


\section{TOMÁS DE AQUINO}

\subsection{Ciencia humana. Cómo se adquiere}

La ciencia creada o humana es la que una persona posee. Y ésta es posible debido a la capacidad racional de todo ser humano. Todo ser humano está dotado por Dios de la luz de la razón, («rationis lumen [...] est nobis a Deo inditum»), y es en base a esa capacidad innata racional recibida de Dios que el ser humano puede conocer y poseer ciencia.

«Praexistunt in nobis quaedam scientiarum semina primae conceptiones intellectus, quae statim lumine intellectus agentis cognoscuntur per species a sensibilibus abstracta, sive sint comple$x a$ (dignitates) sive incomplexa (ratio entis, unius et huiusmodi)». 5

Esta ciencia humana y creada se inicia con una especie de semillas que preexisten en nosotros y que consisten en los primeros conceptos inteligibles, adquiridos con la luz del entendimiento agente por medio de especies abstraídas de las cosas sensibles, especies que pueden ser complejas, como las dignidades, o incomplejas, como la razón de ente o de uno...

Para Tomás de Aquino, toda ciencia se inicia definitivamente en el encuentro del entendimiento agente con las cosas sensibles, «a sensibilibus». Es a partir de las cosas sensibles que la luz del entendimiento agente de inmediato («statim») abstrae las especies inteligibles primigenias de toda ciencia, que pueden ser o complejas, como las dignidades, o incomplejas, como la noción de ente, de uno... Y estos conceptos primigenios del entendimiento producidos a partir inmediatamente de las cosas sensibles son las semillas de las ciencias que preexisten en nosotros, conceptos que pueden ser complejos, como las dignidades («dignitates»), o incomplejos, como la noción de ente, uno, bueno...

Dignitas es la palabra latina equivalente a la griega axioma, usada por Aristóteles para significar «las proposiciones comunes y primeras en las que se basa toda demostración» ( $\tau \alpha$ kotv $\alpha$ $\lambda \varepsilon \gamma o \mu \varepsilon v \alpha \alpha \xi_{1} \omega \mu \alpha \tau \alpha \varepsilon \xi \omega \nu \pi \rho \omega \tau \omega \nu \alpha \pi 0 \delta \varepsilon \iota \kappa v v \sigma 1$, Analíticos Posteriores, I,X, 13-14). El principio de no contradicción es el ejemplo primero de axioma presentado por Aristóteles y el principio de todos los axiomas. Tomás de Aquino presenta como dignidad o axioma la proposición «el todo es mayor que la parte», en la que sù verdad evidente proviene inmediatamente del conocimiento de sus términos y no se precisa ningún término medio para descubrir su verdad. Las dignidades o axiomas son, por tanto, las premisas primeras y más genéricas a partir de las cuales se inicia toda demostración.

Los conceptos incomplejos primeros son también los más genéricos o más universales, de los que derivan todos los demás conceptos. Ejemplos de estas nociones primeras, genéricas y, por tanto, las más universales son ente, uno y bueno, es decir, los trascendentales que se predican de toda realidad existente, sea ésta sustancial o accidental.

Estos axiomas comunes y estas nociones genéricas universales son consideradas por Tomás de Aquino como las semillas o razones seminales preexistentes en nosotros a partir de las cuales todo

5 Tomás de Aquino, «De Magistro», en De Veritate, Quaestiones Disputatae, Turín-Roma, Marietti, 1964, Respondeo, p. 225b. 
otro conocimiento deriva. ${ }^{6}$ Son per se evidentes («per se noti»), como principios fundamentales de todo nuestro conocer, y, por tanto, no precisan de demostración alguna; son conceptos previos a toda demostración. Pero es preciso recordar siempre que estas primeras nociones y axiomas tienen su origen en las especies inteligibles formadas inmediatamente por el entendimiento agente a partir de la abstracción o desmaterialización de las cosas sensibles. Es la realidad externa sensible, en definitiva, el origen último de todo nuestro conocer.

Tenemos, por tanto, en el principio de nuestra ciencia humana un muy pequeño conjunto de conceptos - axiomas y conceptos simples-, evidentes por sí mismos, producidos por nuestro entendimiento agente a partir de las cosas sensibles, y son como semillas o razones seminales a partir de los cuales todo otro conocimiento o ciencia deriva.

Como semillas y razones seminales, estos primeros conceptos, complejos e incomplejos, encierran potencialmente en sí mismos todo otro conocimiento no evidente y derivado de estos principios. Es decir, todo otro conocimiento posterior y derivado de estos principios primeros y universales se encuentra encerrado potencial y activamente en estos principios primigenios. En efecto, los conocimientos posteriores y derivados — que son casi todos— se encuentran en estos conceptos primigenios potencial y activamente, en el sentido de que el principio o potencia intrínseca que estos conceptos encierran tiene potencia suficiente para alcanzar el acto perfecto. El conocimiento humano es considerado por Tomás de Aquino como un despliegue en el que se actualiza y se torna perfecto un conocimiento que se encontraba inicialmente en semilla o potencia y de una manera imperfecta.

Ahora bien, algo preexiste en las cosas naturales de dos maneras: o bien en potencia activa completa, o bien en potencia pasiva. Algo preexiste en potencia activa completa cuando el principio intrínseco tiene potencia suficiente para conducir a un acto perfecto, como sucede en la sanación, pues el enfermo posee naturalmente una potencia activa que conduce a la sanación. ${ }^{7}$ Algo preexiste en potencia pasiva cuando el principio intrínseco no posee potencia suficiente para producir el acto, como sucede cuando el aire se convierte en fuego, ya que esto no se produce por alguna potencia existente en el aire. ${ }^{8}$ El conocimiento posterior y adquirido por los seres humanos se encuentra en los conceptos genéricos y universales primigenios en potencia activa de manera semejante a como la sanación se encuentra en el enfermo también en potencia activa completa. Por eso, la adquisición de ciencia o conocimiento humano es concebido como un desarrollo o despliegue activo a partir de estos primeros conceptos universales evidentes o primeros principios.

\footnotetext{
6 «De Magistro», p. 225: «ex istis principiis omnia principia sequuntur, sicut ex quibusdam rationibus seminalibus».

La comparación de la adquisición de la ciencia humana con el desarrollo activo de las razones seminales es persistente en todo el texto del «De Magistro». Ver, por ejemplo, p. 227a, ad 2um, en donde dice: «in eo qui docetur, scientia praexistebat, non quidem in actu completo, sed quasi in rationibus seminalibus, secundum quod universales conceptiones, quorum cognitio est nobis naturaliter indita, sunt quasi semina quaedam omnium sequentium cognitorum».

7. «De Magistro», art. 1, p. 225b-226a: «Aliquid praeexistit in potentia dupliciter. Uno modo in potentia activa completa; quando scilicet, principium intrinsecum sufficienter potest perducere in actum perfectum, sicut patet in sanatione: ex virtute enim naturale quae est in aegro, aeger ad sanationem perducitur».

8 «De Magistro», art. 1, p. 226a: «alio modo in potentia passiva; quando, sciclicet, principium intrinsecum non sufficit ad educendum in actum, sicut patet quando ex aëre fit ignis; hoc enim non potest fieri per aliquam virtutem in aërem existentem».
} 
El conocimiento humano se inicia a partir del contacto de nuestro entendimiento con la realidad sensible exterior, el cual de una manera inmediata («statim») produce los primeros principios 0 axiomas; todas las formas inteligibles en las que consiste la sabiduría y que se producen como despliegue de los primeros principios no son pura elaboración mental sin contacto alguno con la realidad exterior, sino que son siempre semejanzas de las cosas materiales externas. ${ }^{9} \mathrm{El}$ conocimiento humano echa sus raíces en las cosas materiales sensibles y mantiene siempre con ellas una relación de semejanza; el conocimiento humano no se desvincula jamás de la realidad sensible de las cosas materiales.

\subsection{Maestro y enseñanza}

Este despliegue se produce o bien de una manera autónoma y por la potencia intrínseca naturalmente encerrada en el entendimiento y razón humanas, a lo que se le llama invención y de esa manera el discípulo aprende por sí mismo; o bien, desde fuera, por medio de un agente extrínseco que apoya o ayuda a la razón natural y a esto se le llama disciplina. ${ }^{10} \mathrm{La}$ función del maestro es la de apoyar 0 ayudar a la razón natural o agente intrínseco a que se desarrolle, administrándole aquellas cosas con las que esa potencia pueda actualizarse. Tomás de Aquino compara la potencia activa encerrada en los conceptos genéricos y universales primigenios con la potencia activa encerrada en el enfermo por medio de la cual el enfermo sana. ${ }^{11}$ El enfermo posee una potencia natural que le hace sanar («ex virtute naturali quae est in aegro, aeger in sanitatem perducitur»); de igual manera, el que aprende posee una potencia activa en su entendimiento y razón, recibida de Dios, que le hace adquirir ciencia. Por eso, el enfermo puede frecuentemente curarse solo y el que aprende aprender solo o adquirir ciencia. La naturaleza como potencia activa, tanto en la enfermedad como en la adquisición de la ciencia humana, es el agente principal que hace que el enfermo se cure y el que aprende adquiera ciencia. El médico es para el enfermo y el maestro para el discípulo un agente externo cuyo objetivo es ayudar al agente interno y principal, es decir, al entendimiento humano. Así, el médico ayuda al enfermo confortando su naturaleza y administrándole medicinas, que son como instrumentos que la naturaleza utiliza para curarse; de igual manera, el maestro ofrece al alumno instrumentos o signos con los que la razón del discípulo aprende o conoce en acto lo que previamente conocía sólo en potencia. Existe, sin embargo, una diferencia importante entre el médico y el maestro: el médico puede ayudar a sanar al enfermo, aunque él mismo no esté en acto sano sino enfermo, pero posea el arte de la medicina; el maestro, por el contrario, ve en acto y de una manera completa las nociones y verdades que trata de enseñar al discípulo, el cual tiene de ellas un conocimiento parcial o en sus principios evidentes. El maestro tomista posee no sólo la habilidad discursiva o disciplina con la que puede actualizar las nociones potenciales encerradas en los primeros principios evidentes, sino también la ciencia en acto y de una manera explícita, es decir

9 «De Magistro», art. 1, ad 10m, p. 227a: «Formae enim intelligibiles, ex quibus sapientia consistit, et sunt rerum similitudines, et sunt formae perficientes intellectum».

10 «De Magistro», art. 1, p. 226a: «duplex est modus adquirendi scientiam: unus quando naturalis ratioper seipsum devenit in cognitionem ignotorum; et hic modus dicitur inventio; alius, quando rationi naturali aliquis exterius adminiculatur, et hic modus dicitur disciplina».

11 «De Magistro», art. 1, pp. 225b-226a. 
posee en su entendimiento pasivo actualizadas y explícitas todas las nociones derivadas que intenta causar también de una manera explícita y perfecta en el discípulo. ${ }^{12}$

El paso hacia el conocimiento de lo desconocido es un proceso de la razón por medio del que los principios comunes evidentes se aplican a determinadas materias para alcanzar algunas conclusiones particulares, a partir de las cuales se pasa a otras conclusiones más particulares. ${ }^{13}$ Este discurso de la razón que uno mismo realiza por medio de la razón natural puede uno mostrarlo a otro por medio de signos, a lo que se le llama enseñar. Y la razón natural del discípulo, por medio de lo mostrado por el maestro como un cierto instrumento, alcanza el conocimiento de las cosas desconocidas. Enseñar, por tanto, es mostrar por medio de signos la disciplina o el arte de pasar de los primeros conceptos universales y axiomas a conclusiones particulares desconocidas y de éstas a otras conclusiones particulares igualmente desconocidas. ${ }^{14}$ Pero esta disciplina $o$ arte de pasar de los primeros conceptos universales y axiomas a conclusiones particulares se llama también silogística; por tanto, es fundamental en la docencia mostrar la disciplina de la silogística por la que adquirimos ciencia, «demostratio est syllogismus faciens scire» («D. M.», art. 1, p. 226b).

Cuando alguien muestra a otro lo que no está incluido en los primeros principios evidentes, o no parece estar incluído, no produce ciencia en el otro sino quizás opinión o fe. Ciencia la constituyen las conclusiones que necesariamente se siguen de los primeros principios evidentes y que han de defenderse con total certeza, mientras que han de rechazarse absolutamente como totalmente falsas las proposiciones contrarias a estos primeros principios evidentes. A las demás proposiciones que ni se siguen necesariamente ni son contrarias a los primeros principios universales evidentes, puede libremente asentirse o no.

En conclusión, podemos decir que el maestro enseña por medio de signos sensibles el proceso deductivo, método o disciplina por la cual la razón natural pasa de los primeros conceptos y axiomas universales evidentes a conclusiones particulares encerradas en aquéllas, y de éstas a otras conclusiones particulares igualmente encerradas en los primeros. ${ }^{15}$

\subsection{Ciencia humana actual}

La ciencia humana en potencia, que supone la capacidad racional intelectiva recibida de Dios $\mathrm{y}$, con ella, el conocimiento de los primeros conceptos y axiomas evidentes, es la misma en toda persona humana normal. Pero la ciencia humana actual varía en cada sujeto o persona de acuerdo

12 «De Magistro», art. 1, p. 229a, cuerpo: «Doctrina autem importat perfectam actionem scientiae in docente vel magistro; unde oportet quod ille qui docet vel magister est, habeat scientiam quam in alio causat, explicite et perfecte». También, art. 1, p. 229a, ad 4um: «docens, qui explicite totam scientiam novit [...]». También art. 1, ad 6um: «magister docet inquantum actu scientiam habet».

13 «De Magistro», art. 1, p. 226a: «Processus rationis pervenientis ad cognitionem ignoti in inveniendo est ut principia communis per se nota applicet ad determinatas materias, et inde procedat in aliquas particu-lares conclusiones, et ex his in alias».

14 «De Magistro», art. 1, p. 226a: «unus alium docere dicitur, quod istum discursum rationis, quem in se facit rationi naturali, alteri exponit per signa et sic ratio naturalis discipuli, per huiusmodi sibi proposita, sicut per quaedan instrumenta, pervenit in cognitionem ignotorum».

15 «De Magistro», art. 3, p. 231b: «ex parte principiorum potest [homo] docere [...] deductionem conclusionum ex principiis sub signis sensibilibus proponendo». 
con la disciplina o habilidad racional que tenga para desplegar toda la potencialidad activa encerrada en los primeros conceptos y axiomas genéricos y evidentes. ${ }^{16} \mathrm{La}$ ciencia humana en acto de cada sujeto o persona es el conjunto de formas o especies inteligibles adquiridas por medio de un proceso por el que se actualizan las potencias activas encerradas en los primeros conceptos y axiomas universales y evidentes. ${ }^{17}$ La ciencia humana actual de cada sujeto está constituida por los primeros conceptos y axiomas evidentes y universales, y por todo otro concepto o proposición adquiridos y derivados de los primeros por medio de una relación lógico-necesaria. La capacidad de derivar con necesidad lógica conceptos y verdades a partir de los primeros conceptos evidentes y universales radica en el entendimiento humano agente, pero puede mejorarse con el ejercicio o práctica, alcanzando lo que Tomás de Aquino llama disciplina. Y en la adquisición de la disciplina o habilidad de desplegar las potencialidades activas de los primeros conceptos y axiomas tiene mucha influencia la docencia del maestro, como agente externo y mediato; en efecto, por medio de ejemplos y ejercicios, es decir, signos, puede lograr desde afuera que el discípulo adquiera el hábito de utilizar la disciplina silogística por la que actualizamos los conceptos y verdades potenciales encerrados en los primeros conceptos y axiomas. El entendimiento humano, dice el Aquinate, es colativo, es decir, a partir de unas verdades llega a descubrir otras; por tanto, el entendimiento humano no tiene la misma relación con respecto a todas las formas o especies inteligibles. Algunas, primeras y evidentes, las posee en acto desde el momento mismo en que es racional; pero otras están implícitas en estas primeras y no llega a descubrirlas sino después de un proceso de despliegue de las potencialidades de las primeras, que se produce por medio del ejercicio de la razón y el entendimiento, ejercicio que entraña una habilidad y un hábito, sin el cual el entendimiento agente no se encuentra sólo en potencia accidental sino incluso en potencia esencial con respecto a las nuevas formas inteligibles. ${ }^{18}$ Es decir, el maestro es, en esta concepción tomista, necesario para desarrollar en toda su plenitud la capacidad colativa del entendimiento por la que de unas verdades universales conocidas alcanza otras verdades menos universales, nuevas y desconocidas. Sin ayuda del maestro, el discípulo que no posea todavía el hábito deductivo está en potencia esencial con respecto a los nuevos conceptos o verdades. El discípulo, en opinión del Aquinate, sin ayuda del maestro exterior puede, por la capacidad de su propio entendimiento agente, adquirir nuevas nociones o formas inteligibles deduciéndolas de los primeros principios evidentes. Pero con la ayuda del maestro la adquisión de las nuevas formas inteligibles o verdades se producirá con mayor celeridad, con más seguridad y el número de formas inteligibles o verdades será más numeroso. El maestro, poseedor ya del hábito deductivio, desempeña, por tanto, una función esencial en el proceso por el que

16 «De Magistro», art. 1, p. 227a, ad 10um: «Alio modo secundum esse quod habet in subiecto; et sic per accidens mutatur, subiecto mutato de potentia habente sapientiam in actu habens».

17 «De Magistro», art. 1, p. 227a, ad 10um: «formae enim intelligibiles, ex quibus sapientia consistit [...]». También, p. 227b, ad 11um: «formae intelligibiles, ex quibus scientia per doctrinam accepta constituitur, inmediate per intellectum agentem, sed mediate per eum qui docet».

18 «De Magistro», art. 1, p. 227b, ad 12um: «Sed potentia intellectiva cum sit collativa, ex quibusdam in alia devenit; inde non se habet aequaliter ad omnia intelligibilia consideranda; sed statim quaedam videt, ut quae sunt per se nota, in quibus implicite continentur quaedam alia quae intelligere non potest nisi per officium rationis ea quae in principiis implicite continentur, explicando; unde ad huiusmodi cognoscenda, antequam habitum habeat, non solum est in potentia accidentali, sed etiam essentiali: indiget enim motore, qui reducat eum in actum per doctrinam [...]». 
el discípulo adquiere la habilidad y el hábito de deducir, a partir de nociones y axiomas evidentes, nuevas nociones y verdades encerradas en las primeras. El maestro excita al discípulo por medio de palabras y ejercicios verbales silogísticos a que descubra por sí mismo las nuevas formas inteligibles y verdades encerradas en las primeras nociones y axiomas. Una vez adquirido el hábito deductivo, el discípulo podrá facilmente, él mismo, desplegar y descubrir nuevas nociones y verdades.

La razón humana y, en concreto, el entendimiento agente es la causa efectiva próxima de la ciencia humana actual, y el maestro es el agente externo y mediato que ayuda por medio de signos sensibles al entendimiento en la perfección de la ciencia, «lumen rationis coadjuvans ad scientiae perfectionem per ea quae exterius proponit» («D.M.», art. 1, p. 227a, ad 4um y ad 9um). Los signos sensibles o palabras, orales y escritas, utilizadas por el maestro docente son causas de ciencia de manera semejante a como lo son las cosas sensibles a partir de las cuales el entendimiento agente abstrae las formas o especies inteligibles; incluso las palabras del maestro son causas más cercanas de la ciencia que las cosas sensibles en la medida en que son signos de intenciones o formas inteligibles. ${ }^{19} \mathrm{Si}$ quisiéramos definir las causas en este proceso de conocer y de descubrir nuevos conceptos y verdades, diríamos que las cosas sensibles y las palabras del maestro son causas instrumentales que ayudan al entendimiento agente a formar conceptos y verdades; los conceptos y axiomas primitivos vendrían a ser la causa material a partir de la que el entendimiento elabora las nuevas nociones o especies inteligibles; la razón humana y, en concreto, el entendimiento agente sería la causa eficiente inmediata productora 0 , mejor, descubridora de los nuevos conceptos y verdades como causas formales; el maestro vendría a ser un agente exterior, el cual con su ayuda logra que el entendimiento agente realice con perfección, seguridad y rapidez su operación inteligible; y la causa final sería la ciencia misma adquirida.

La certeza o verdad científica que debe acompañar a las nuevas nociones inteligibles proviene, no de la autoridad del maestro, sino de los principios cognoscitivos — conceptos y axiomas evidentes- de los que lógica y necesariamente se derivan. Por eso, las nuevas nociones serán ciertas y absolutamente verdaderas sólo si pueden resolverse en los primeros principios evidentes. En última instancia, la certeza científica es una propiedad proveniente de la luz de la razón recibida interiormente de Dios por medio de la cual Dios nos habla. ${ }^{20}$

19 «De Magistro», art. 1, p. 227b, ad 11um: «Unde ipsa verba doctoris audita vel visa inscripta, hoc modo se habent ad causandum scientiam in intellectu sicut res quae sunt extra animam, quia ex utrisque intellectus intentiones intelligibiles accipit; quamvis verba doctoris propinquius se habeant ad causandum scientiam quam sensibilia extra animam existentia inquantum sunt intelligibilium intentionum».

20 «De Magistro», art. 1, p. 227b, ad 13um: «certitudo scientiarum tota oritur ex certitudine principiorum; tunc enim conclusiones per certitudinem sciuntur, quando resolvuntur in principia. Et ideo, quod aliquid per certitudinem sciatur, est ex lumine rationis divinitus interius indito, quo in nobis Deus loquitur; non autem ab homine exterius docente, nisi quatenus conclusiones in principia resolvit, nos docens $[\ldots]$... 


\section{CONCLUSIÓN}

Notables e importantes diferencias existen entre el De Magistro agustiniano y el «De Magistro» tomista. Señalaremos algunas:

\section{A) Diferente concepto de ciencia}

Para Agustín la verdadera ciencia es la ciencia de las realidades o verdades inteligibles. Esta ciencia se desarrolla en el interior del hombre o en «el hombre interior» por medio de un proceso intuitivo inteligible y racional. No tiene contacto alguno con el mundo de las cosas materiales; al contrario, entraña un proceso de superación y alejamiento del mundo material y del cuerpo propio. Tal proceso de superación del mundo material y del cuerpo propio se alcanza por medio de un control y dominio perfecto de las facultades de la mente o del alma sobre las facultades sensibles del cuerpo, especialmente sobre la libido o concupiscencia del cuerpo; el dominio de la «buena voluntad» sobre la «mala voluntad». La ciencia intuitiva inteligible y racional agustiniana dice una relación directa y proporcional con la buena voluntad, con la virtud o santidad en la que se desarrolla el «hombre interior» y con él la ciencia o sabiduría. El fín último de esta ciencia agustiniana es la unión mística con la Divinidad, descrita como luz interior y como Verdad eterna e inmutable.

La ciencia humana tomista se enraíza siempre en el mundo de las cosas materiales, de donde provienen inmediatamente, «statim», los primeros principios y axiomas evidentes, y, de una forma mediata, todas las formas inteligibles derivadas que son semejanzas de las cosas materiales. El entendimiento agente humano, como facultad colativa o discursiva, es la causa eficiente de esta ciencia tomista. La ciencia del maestro tomista es entendida como un despliegue deductivo o actualización de todas las potencialidades encerradas en los primeros principios o axiomas evidentes. Esta ciencia tomista no parece tener relación directa e inmediata con la santidad de la persona que la posee, sino más bien con su habilidad deductiva o disciplina.

B) Distintas son también las nociones de maestro en Agustín y Tomás de Aquino:

«Maestro», para Agustín, es el que muestra a los ojos, a algún sentido del cuerpo o a la mente aquello que se quiere conocer (D.M.,11.36). Puede haber y hay muchos maestros de la ciencia sensible y práctica que utilizan el método audiovisual. Pero no puede haber maestro humano alguno capaz de mostrar a la intuición inteligible del hombre interior una sola verdad eterna e inmutable. En el hondón del alma, alcanzado por un proceso ascético de dominio y control de la buena voluntad sobre la libido y la concupiscencia del cuerpo, sólo enseña Cristo o la sabiduría sempiterna. La palabras o signos sensibles externos sólo pueden estimular de una manera mediata el recogimiento y la introspección como requisitos necesarios para el desarrollo del «hombre interior» en el que se desarrolla la verdadera ciencia agustiniana. El maestro agustiniano tiene que ser esencialmente bueno y, por eso, no podemos encontrarlo en la tierra; el único maestro de todo se encuentra en los cielos, «vere scriptum est autoritate divina ne nobis quemquam magistrum dicamus in terris, quod unus omnium magistrum in coelis est y es Cristo o Dios» (D.M., 14,46).

Tomás de Aquino distingue dos maestros: uno principal e interior y otro secundario y externo. El maestro principal e interior es Dios, quien ha dotado al ser humano del entendimiento o de la capacidad discursiva por medio de la cual adquiere la ciencia. Pero el hombre también puede con- 
siderarse como maestro, secundario y externo, el cual con palabras y signos sensibles ayuda a desarrollar la habilidad discursiva o disciplina del discípulo y con ella a aumentar el conjunto de especies inteligibles en el entendimiento, lo que constituye el contenido de la ciencia humana. El maestro tomista posee en acto y de una manera explícita toda la ciencia que va a enseñar, es decir, la habilidad discursiva o disciplina y la totalidad de las nociones o verdades, primeras y derivadas, que va a enseñar.

C) Son también distintas las concepciones agustiniana y tomista del discípulo:

$\mathrm{Si}$, para Agustín, Dios o la Verdad es el único maestro interior, todos los seres humanos podemos ser sus discípulos, «unus omnium magister in coelis est» (D.M., 14,45). Todos podemos ser discípulos de la Verdad interior, pero en realidad son discípulos de la Verdad sólo quienes pueden ver interiormente, «quisquis autem cernere potest, intus est discipulus veritatis» (D.M., 13,41). Es decir, sólo quienes poseen la «buena voluntad» para obrar rectamente o quienes han desarrollado el hombre interior de manera que puedan ser capacer de intuir mentalmente en su interior la luz de la Verdad, sólo ésos, son los auténticos discípulos de la verdad y de Cristo. La buena voluntad para obrar rectamente es el requisito indispensable para ser discípulo de la Verdad o de Cristo; y tanto más ciencia interior alcanzará una persona cuanto más rectamente obre.

El discípulo, para Tomás de Aquino, posee la ciencia humana sólo en parte, a saber, en sus principios axiomas primeros y evidentes o en sus razones seminales («D.M.», c, p. 229a). El aprendizaje consistirá, precisamente, en adquirir la habilidad o la doctrina discursiva que despliegue al máximo y actualice las nociones inteligibles derivadas encerradas en ellas. $Y$ en este proceso de adquisición de la doctrina o arte discursivo desempeña el maestro externo un papel importante. Puede el discípulo por sí mismo y por invención adquirir nuevas nociones inteligibles o verdades; pero, con la ayuda del maestro y con el hábito y la disciplina discursiva, adquirirá más verdades y con mayor rapidez y seguridad.

Antonio Pérez-Estévez Instituto de Filosofía

CSIC

Pinar, 25

E-28006 MADRID 